\title{
Ultrasound for Critical Care Physicians: A Tempting Dilemma
}

\author{
Issam Marzouk MD \\ Lana Melendres MD \\ Michel Boivin MD \\ Division of Pulmonary, Critical Care and Sleep \\ Department of Medicine \\ University of New Mexico School of Medicine \\ MSC 10-5550 \\ Albuquerque, NM 87131 USA
}

A 46 year old woman presented with progressive severe hypoxemia and a chronic appearing pulmonary embolus on chest $\mathrm{CT}$ angiogram to the intensive care unit. The patient was hemodynamically stable, but had an oxygen saturation of $86 \%$ on a highflow $100 \%$ oxygen mask. The patient had been previously investigated for interstitial lung disease over the past 2 year, this was felt to be due to non-specific interstitial pneumonitis. Her echocardiogram findings are as presented below (Figures 1 and 2).

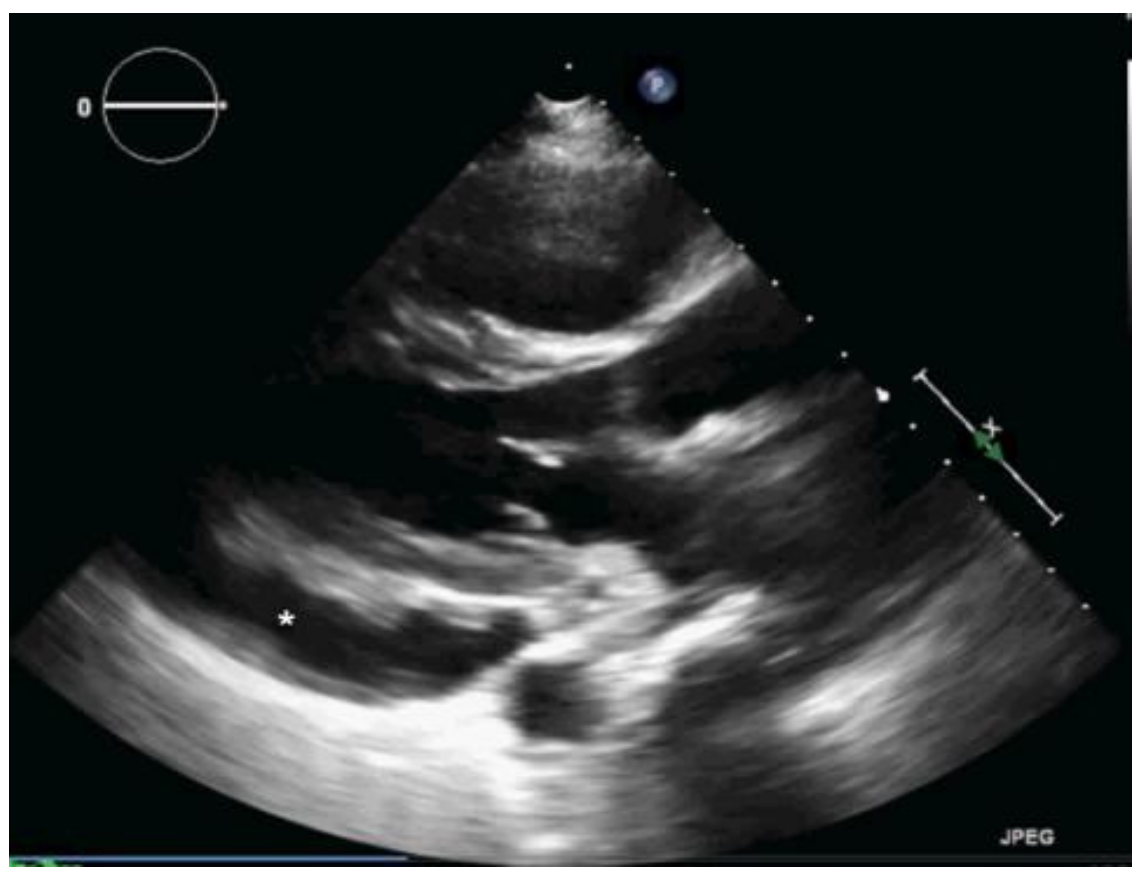

Figure 1. Parasternal long axis view. 


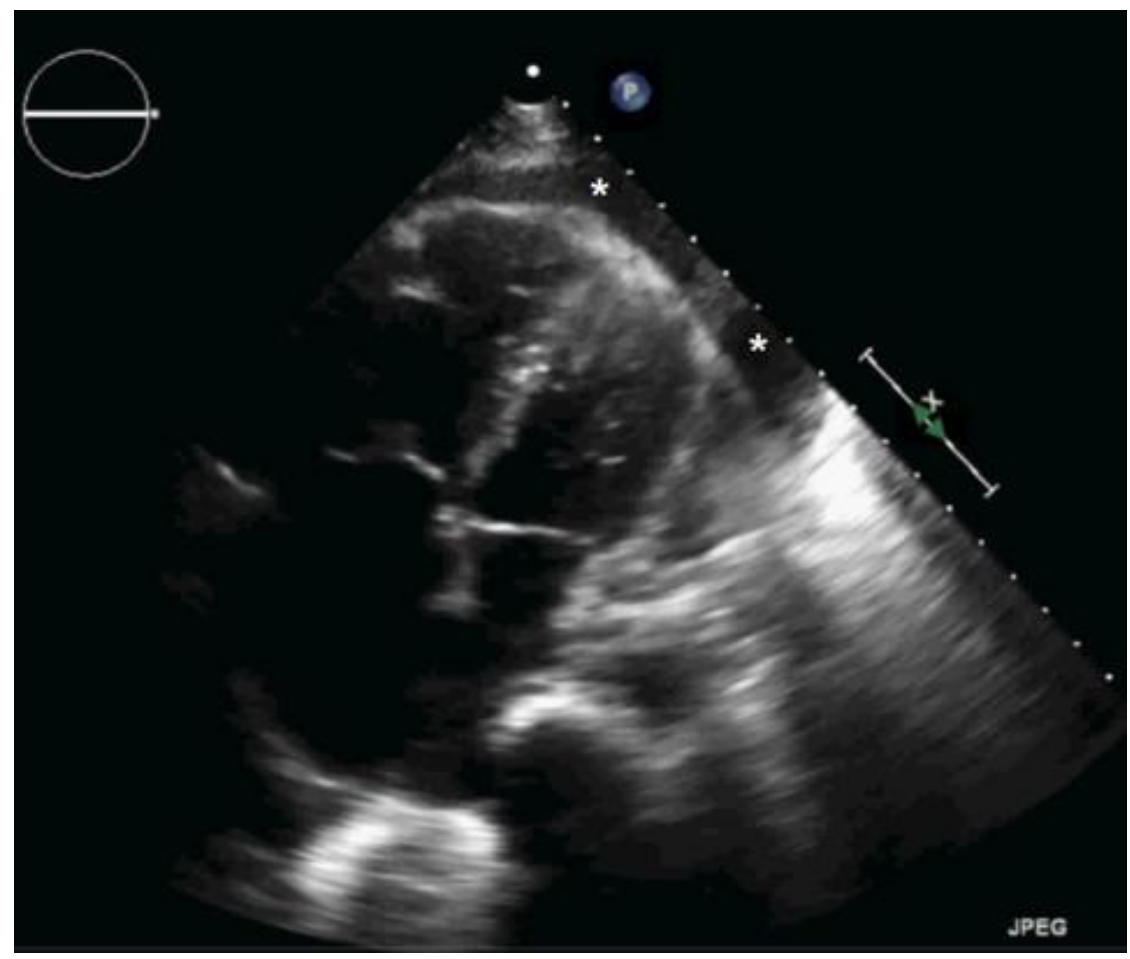

Figure 2. Apical four chamber view.

The patient had refractory hypoxemia despite trials of high flow oxygen and noninvasive positive pressure ventilation. She had mild symptoms at rest but experienced severe activity intolerance secondary to exertional dyspnea. Vitals including blood pressure remained stable and normal during admission and the patient had a pulsus paradoxus measurement of $<10 \mathrm{mmHg}$. She had previously had an echocardiogram 6 months before that revealed significant pulmonary hypertension.

What would be the most appropriate next step regarding management of her echocardiogram findings?

1. Diagnostic pericardiocentesis

2. Urgent therapeutic pericardiocentesis

3. Avoid pericardiocentesis at this time

4. Urgent thrombolysis with tissue plasminogen activator 


\section{Correct! \\ 3. Avoid pericardiocentesis at this time}

The patient had a large pericardial effusion (as demonstrated by asterisks) in Figure 1 and Figure 2. Furthermore, the patient had a massively dilated right ventricle (RV). The $\mathrm{RV}$ is significantly larger than the left ventricle in the apical 4-chamber view. As demonstrated in Figure 3 , the velocity of the jet of tricuspid regurgitation suggests a pressure difference of $57 \mathrm{mmHG}$ between right atrium and pulmonary artery.

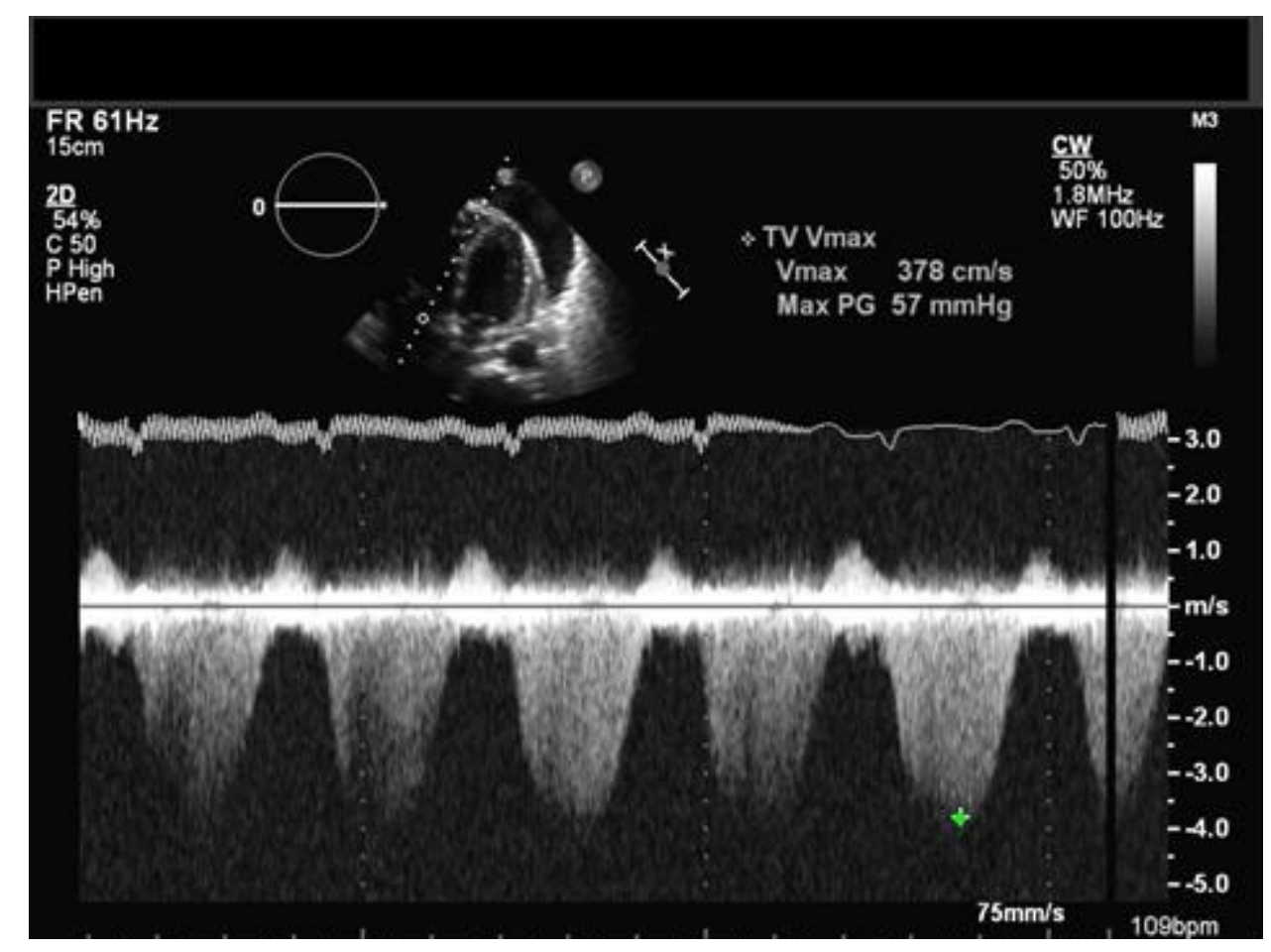

Figure 3. Spectral continuous-wave Doppler interrogation through the tricuspid valve demonstrating a peak velocity of $3.78 \mathrm{~m} / \mathrm{s}$.

The patient had a dilated non-variable inferior vena cava (not shown) which would estimate right atrial pressure at a minimum of $15 \mathrm{mmHG}$, and thus an estimated systolic pulmonary arterial pressure of $72 \mathrm{mmHG}$. These findings together suggest the patient likely has severe pulmonary hypertension and RV failure. While the patient did have evidence of past thromboembolism on the CT angiogram, it was not felt to be acute. The pulmonary artery pressures were only slightly elevated from 6 months previous.

Many patients with advanced pulmonary hypertension develop pericardial effusions related to elevated right sided heart pressures, this finding is associated with a poorer prognosis in patients with pulmonary arterial hypertension (1). Furthermore, diagnosis of 
pericardial tamponade is complicated in these patients in that dilated IVC and exaggerated respiratory variation of mitral and tricuspid inflows is common to both pericardial tamponade and pulmonary hypertension (2). Volume overloaded and hypertrophied right sided chambers are also less likely to demonstrate collapse (2). The lowest pressure chamber in right heart failure is often the left atrium, but due to its extrapericardial nature in many patients, it may not demonstrate evidence of collapse. A diagnosis of pericardial tamponade is exceedingly challenging in this population.

Additionally, pericardiocentsis in patients with severe pulmonary arterial hypertension is associated with very poor outcomes. A recent case series by Hemnes et al. (3), showed that 5 of 5 consecutive patients with severe pulmonary arterial hypertension undergoing pericardial drainage died within 24 hours. The outcomes of pericardial effusions in patients with group 3 and 4 pulmonary hypertension (Dana Point classification) as this patient had are much less clear. Extreme caution is advised when considering pericardiocentesis in this population. This patient's pericardial effusion was managed conservatively.

\section{References}

1. Fenstad ER, Le RJ, Sinak LJ, Maradit-Kremers H,et al. Pericardial effusions in pulmonary arterial hypertension: characteristics, prognosis, and role of drainage. Chest 2013;144(5):1530-8. [CrossRef] [PubMed]

2. Plotnick GD, Rubin DC, Feliciano Z, Ziskind AA. Pulmonary hypertension decreases the predictive accuracy of echocardiographic clues for cardiac tamponade. Chest 1995;107(4):919-24. [CrossRefl [PubMed]

3. Hemnes AR, Gaine SP, Wiener CM. Poor outcomes associated with drainage of pericardial effusions in patients with pulmonary arterialhypertension. South Med J. 2008;101(5):490-44. [CrossRef] [PubMed] 